\title{
Erosion of fine sediments from a rough bed
}

\author{
Michele Trevisson ${ }^{1, *}$ and Olivier Eiff $^{1}$ \\ ${ }^{1}$ Institute for Hydromechanics, Karlsruhe Institute of Technology, Karlsruhe, Germany
}

\begin{abstract}
Gravel beds in river systems represent important aquatic habitats, which may be endangered by the introduction of large amounts of fine sediments. In order to better understand the interaction between fine sediments and coarse immobile beds in sediment supply-limited systems, a series of flume experiments was conducted. The main goal was to determine under what conditions erosion stops. The experiments were performed over a bed of regularly arranged spheres. Plastic particles were taken as sediment and the erosion was investigated under uniform flow conditions for variable bed shear stress conditions just above critical conditions. The system was observed to behave in two different ways: with higher bed shear stress fine sediments were completely washed out, whilst with lower stress the sediment bed reached a stable level just above the equator of the spheres.
\end{abstract}

\section{Introduction}

Sediment supply-limited environments characterize many river systems, where the availability of sediments is reduced due to land use practices or dam regimentations. For the latter, sediment supply is connected to the release of limited amounts of sediments due to dam removal or dam flushing [1]. As a consequence, the gravel beds are characterized by travelling fine sediment clouds, which tend to fill up the bed interstices and the river pool systems, leading to a reduction of fish habitats [2]. The release of fine sediments leads to a change in the grain size distribution of the river, which is characterized, as a result, not only by gravel, immobile for most of the flow conditions, but also easily mobilized fine sediments [3]. Since sediment transport is strongly influenced by the grain sizes involved, classical theories for the prediction of sediment transport for beds composed of uniform sediments, such as [4-6], cannot directly be applied any more. Only recently, models for sand/gravel systems have started to be developed [7-10], but the interaction between fine sediments over a rough immobile substrate is still not well understood.

The complexity of these systems lies in the fact that the transport depends not only on the absolute grain size of the sediments, but also on the relative size of the whole sediment fractions present on the immobile bed roughness characteristics [11]. On one hand, the large immobile roughness elements like gravel are taken to be able to shield the fine sediment, thereby reducing their mobility [12]. On the other hand, they can induce local flow perturbations which can increase the local shear and therefore enhance or induce sediment transport [3]. Raupach [13], in the context of atmospheric boundary layers canopies, introduced the concept of "shear-stress partitioning". With this concept, only part of the total shear stress acts

\footnotetext{
*e-mail: michele.trevisson@kit.edu
} 
on the base of the bed, i.e. on the finer sediments, the rest on the protruding roughness elements. The model is controlled by two parameters, the protrusion of the immobile roughness elements, expressed as roughness density (ratio between the frontal area of the roughness elements and the planar reference area) and the spacing between roughness elements [9]. In the field of aeolian sand transport over rough surfaces, $[14,15]$ have shown that the erosion rates of sand under sediment supply-limited conditions reduce in time as the roughness elements protrusion increases, in general accordance with shear stress partitioning, although an initial enhancement of transport, when the elements first appear, has also been observed, suggesting the influence of turbulence induced vortices [14]. In river flows, [3] has observed similar behaviours, but with sediment feeding. No studies have shown, whether protruding roughness elements can stop sediment transport of the finer fractions in sediment supply limited conditions.

Sediment transport in sediment-supply limited environments is furthermore complicated by the possible formation of bed-forms. Under sediment-supply limited conditions, two different types of bed-forms can develop: barchans and ridges [16], caused by secondary currents [17]. The presence of these bed-forms can greatly influence the fine sediment coverage of the bed and interacts with the shear stress distribution over the bed [18]. For example, the flow over ridges is characterized by upward-flow with lower shear stress, while the flow over the troughs witnesses down-flow with higher shear stress $[19,20]$, leading to higher and lower sediment transport, respectively. However, bed-forms with immobile roughness elements have not yet been studied.

This work aims at analysing the erosion of fine sediments in a coarse immobile substrate, made of a regular array of spheres, under sediment supply-limited conditions. An experimental approach has been chosen, in order to determine whether a static equilibrium of the bed in the Bagnold sense [21] can be achieved, i.e. a condition characterized by a constant bed level. For sediment supply-limited conditions, this implies the suppression of fine sediment transport. The experiments were conceived so as to analyse the morphodynamics of the bed, by identifying trends in the erosive behaviour.

\section{Experimental design and methodology}

\subsection{Experimental facilities and sediment modelling}

The experiments were performed in a $9 \mathrm{~m}$ long, $30 \mathrm{~cm}$ wide and $32 \mathrm{~cm}$ high recirculating and tilting flume at the Institute of Hydromechanics at KIT. A sediment trap is used to collect the fine sediments at the outlet of the flume and no upstream sediment supply is installed. A calibrated venturimeter and a limnimeter are used to measure, respectively, the discharge and the water levels.

Polyurethane spheres $2 \mathrm{~cm}$ in diameter were used to construct a regular and well-defined bed. The spheres were glued in a staggered symmetric pattern, with a frontal density $\lambda_{f}=$ $A_{f} / A_{p}=0.528$, on glass plates and positioned on the bottom of the flume (figure 1).

Fine sediment is modelled with plastic granulate, made of a mixture of urea, melamine and phenol with a specific weight $\rho_{S}$ of $1.49 \mathrm{~g} / \mathrm{cm}^{3}$ and a grain size range of $1.4-2.2 \mathrm{~mm}$, a $d_{50}$ equal to $1.7 \mathrm{~mm}$ and $\sigma_{g}=d_{84} / d_{50}$ of 1.13 , which classifies the plastic granulates as uniform according to [22].

For the onset of each of the experiments, the spheres were covered with a layer of plastic granulates $3 \mathrm{~cm}$ thick (i.e. $1 \mathrm{~cm}$ above the crest), uniformly distributed over the whole length of the flume. The water flow rate was then carefully increased to the final working conditions, in order to avoid an abrupt flushing of the sediments and generate reproducible flow conditions. 


\subsection{Data collection and flow setup}

The design of the experiments aims at analysing the erosive behaviour of the bed and identifying trends in its time evolution. To do so, a $90 \mathrm{~cm}$ long area of the bed, $3.70 \mathrm{~m}$ downstream of the inlet, was recorded with a Prosilica GE 1650 camera with a resolution of 1600x1200 pixel and equipped with a Sigma $24 \mathrm{~mm}$ f1.8 EX DX lens. The camera was positioned perpendicularly to the bed at a height of $2.10 \mathrm{~m}$ (figure 1). The images were recorded with a resolution of $17.14 \mathrm{pixel} / \mathrm{cm}$ at a sampling frequency of $0.1 \mathrm{~Hz}$. Owing to the fact that the Froude numbers were well below 1, no surface-waves formed and it was possible to record directly through the water surface.

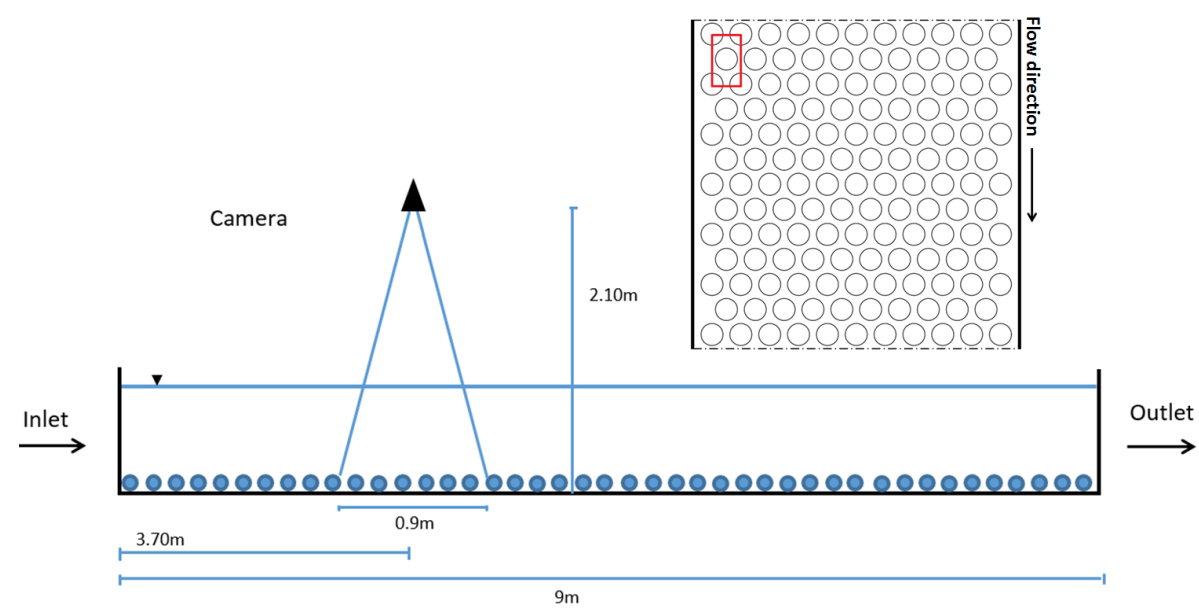

Figure 1. Experimental setup and, in the upper right corner, top view of the flume with the staggered pattern of the spheres (a typical pattern is indicated)

A total of five experiments were performed and the uniform flow conditions were chosen to guarantee a Shields number $\theta=u_{*}^{2} /\left(g \Delta d_{50}\right)$ (where $u_{*}$ is the shear velocity and $\Delta=0.49$ is the relative density) above the uniform threshold $\left(\theta_{t, u}=0.031\right)$. Two parameters were varied in the experiments, the Shields number (by adjusting the slope and the water depth) and the flow aspect ratio $A R=W / H$ (where $H$ is the water depth and $W$ is the width of the flume), in order to determine their influence on the evolution of the bed (see Table 1). Experiments $1,2,3$ were performed with same AR, while experiments $1,4,5$ with same $\theta$ (see Table 1 ). Velocity measurements were performed via particle image velocimetry (PIV), downstream of the measurement area. These show that the flow is fully developed.

Table 1. Flow parameters of the experiments: $Q$, flow rate; $i$, slope; $H$, water depth; $R_{h}$, hydraulic radius; $A R$, aspect ratio; $U$, bulk velocity; $F r=U / \sqrt{g H}$, Froude number; $u_{*}=\sqrt{g R_{h} i}$, total shear velocity; $\Theta$, Shields parameter; $R e^{*}=u_{*} d / v$, sedimentological Re number; $R e=H U / v$, Reynolds number; $T$, time duration of the experiment

\begin{tabular}{|c|c|c|c|c|c|c|c|c|c|c|c|c|}
\hline & $\begin{array}{l}Q \\
{[1 / \mathrm{s}]}\end{array}$ & $\begin{array}{l}i \\
{[\%]}\end{array}$ & $\begin{array}{l}H \\
{[\mathrm{~cm}]}\end{array}$ & $\begin{array}{l}R_{h} \\
{[\mathrm{~cm}]}\end{array}$ & $A R$ & $\begin{array}{l}U \\
{[\mathrm{~cm} / \mathrm{s}]}\end{array}$ & $F r$ & $\begin{array}{l}u_{*} \\
{[\mathrm{~cm} / \mathrm{s}]}\end{array}$ & $\Theta$ & $R e$ & $R e^{*}$ & $\begin{array}{l}T \\
{[\mathrm{~h}]}\end{array}$ \\
\hline Exp 1. & 5 & 0.16 & 6.5 & 4.5 & 4.6 & 25.6 & 0.32 & 2.7 & 0.086 & 17000 & 45 & 71 \\
\hline Exp 2. & 7.4 & 0.25 & 6.84 & 4.7 & 4.4 & 36.1 & 0.44 & 3.4 & 0.141 & 25000 & 58 & 70 \\
\hline Exp 3. & 6.1 & 0.20 & 6.85 & 4.7 & 4.4 & 29.7 & 0.36 & 3.0 & 0.113 & 20000 & 52 & 44 \\
\hline Exp 4. & 3 & 0.23 & 4.5 & 3.5 & 6.7 & 22.2 & 0.33 & 2.8 & 0.096 & 10000 & 48 & 67 \\
\hline Exp 5. & 9.4 & 0.12 & 10.26 & 6.1 & 2.9 & 30.5 & 0.30 & 2.7 & 0.088 & 31000 & 46 & 66 \\
\hline
\end{tabular}


From the images the area of the protruding spheres $A_{p}$ was extracted and the equivalent diameter $D_{e q}=\sqrt{A_{p} 4 / \pi}$ determined. From $D_{e q}$ the frontal area of the protruding spheres was calculated. This allowed to determine the mean roughness density of the bed, $\lambda_{f}$, over $87 \%$ of the width. $\lambda_{f}$ is the ratio between the frontal area of the total number of the protruding spheres $A_{f}$ and a planar reference area $A_{r}$ multiple of the reference pattern of figure 1 . The roughness density enables to quantitatively characterize the roughness of the bed as the bed erodes.

\section{Experimental results}

\subsection{Qualitative description of the evolution of the bed: a bed-form analysis}

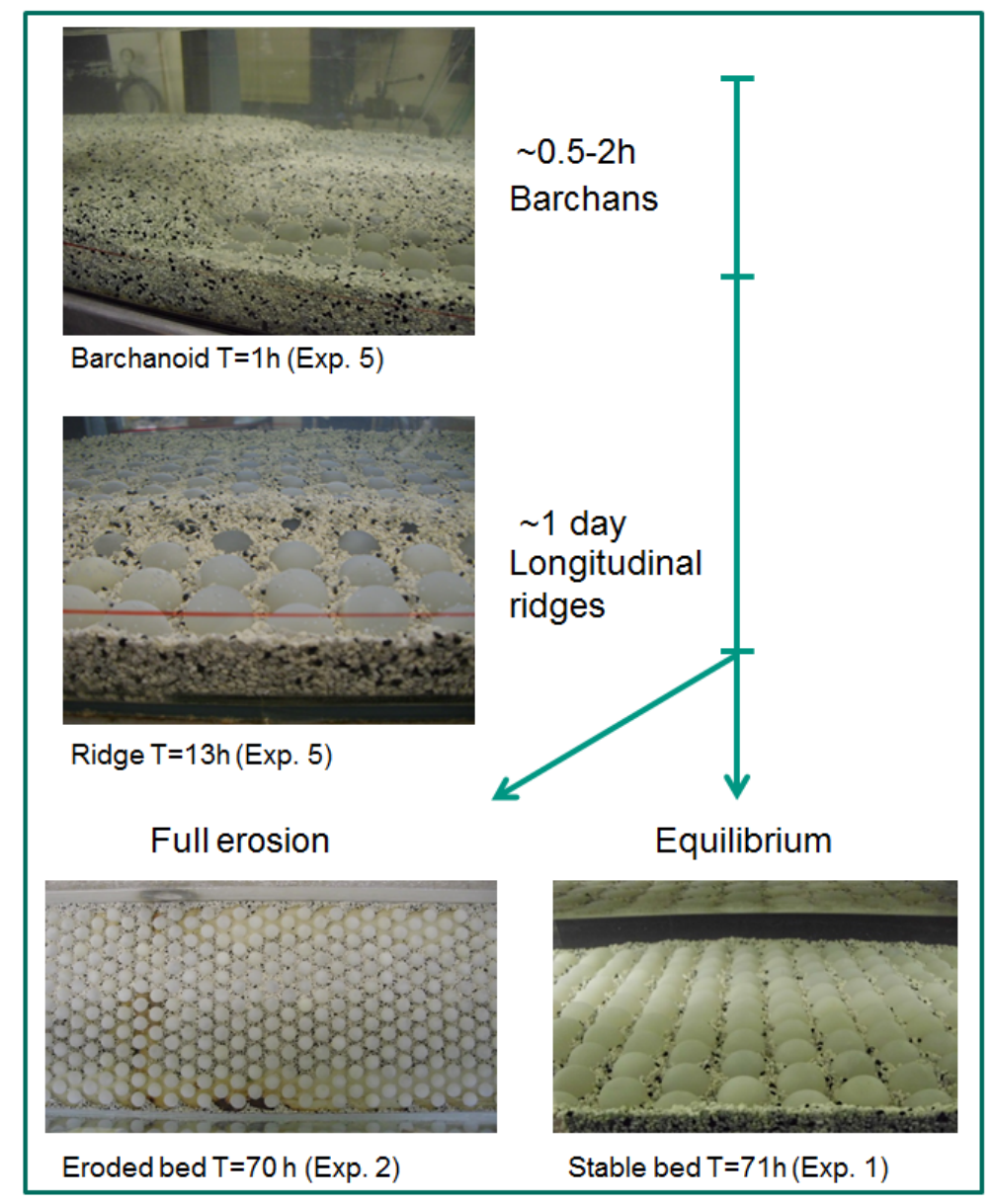

Figure 2. Evolution of the bed in time

During the first phase of all experiments between $\mathrm{t}=0.5-2 \mathrm{~h}$, barchanoids (intermediate bed-forms between dunes and barchans) developed on the bed (see Figure 2) with different heights and celerities (see Table 2). During Exp. 1 barchanoids of $1 \mathrm{~cm}$ height were observed. In the case of Exp. 2 and 3 with same water depth but higher shear stress than in 
Exp. 1, the barchanoids monitored were double as high $(2 \mathrm{~cm})$. Higher shear stress leads to higher sediment transport rates and, therefore, to higher barchanoids. It can be shown, that the height of a dune is proportional to the Shields parameter [23, 24] and evidently the barchanoids behave like dunes.

Table 2. Water depth $H$, shear velocity $u_{*}$, height of the dunes $h_{\text {dune }}$, time at which the barchanoids disappear $T_{b}$ and number \# of ridges for the different experiments

\begin{tabular}{llllll}
\hline & $\begin{array}{l}H \\
{[\mathrm{~cm}]}\end{array}$ & $\begin{array}{l}h_{\text {dune }} \\
{[\mathrm{cm}]}\end{array}$ & $\begin{array}{l}u_{*} \\
{[\mathrm{~cm} / \mathrm{s}]}\end{array}$ & $\begin{array}{l}T_{b} \\
{[\mathrm{~h}]}\end{array}$ & \# ridges \\
\hline Exp. 1 & 6.5 & 2.7 & 1 & 2.34 & 1 \\
Exp. 2 & 6.84 & 3.4 & 2 & 0.55 & 1 \\
Exp. 3 & 6.85 & 3.0 & 2 & 0.92 & 1 \\
Exp. 4 & 4.5 & 2.8 & 0.5 & 1.57 & 2 \\
Exp. 5 & 10.26 & 2.7 & 3 & 1.86 & 1 \\
\hline
\end{tabular}

Exp. 4 with same shear velocity but lower water depth than Exp. 1 displayed lower barchanoids than $0.5 \mathrm{~cm}$. In Exp. 5 with same shear velocity but higher water depth than Exp. 1 and 4, barchanoids of $3 \mathrm{~cm}$ developed. The water depth can be therefore seen as a limiting factor for the height of the barchanoids, as for dunes: the higher the water depth, the higher the dunes which can develop, given a certain shear stress. As stated by [25], high water levels allow a freer development of the dunes which will reach the maximum height, when the flow, being accelerated between the water surface and the dune, reaches a critical suspension velocity.

Since the system was under sediment supply-limited conditions, the barchanoids disappeared within 0.5-2 hours, as a consequence of the depletion of the layer of sediments above the top of the spheres. At this point, a formation of ridges was observed in all experiments (see figure 2). The bed topography for all experiments in Table 2 was characterized by areas of higher sediment level (side-wall accumulations and ridges) alternating with lower eroded areas. It was observed that the number of ridges was correlated to the aspect ratio AR: for AR lower than 5 (Exp. 1, 2, 3, 5) only one ridge developed in the middle of the flume, while for AR bigger than 5 (Exp. 4) two narrow ridges appeared. Unlike the barchanoids, the ridges remained on the bed for a longer period. This is connected to the fact, that the secondary flows, which generate the ridges, also induce sediment transport fluxes directed towards the ridge [17]. The ridge was therefore continuously supplied with new sediment transported away from the eroded areas. Nevertheless, due to eventual reduction of sediment availability from upstream and the preferential longitudinal sediment transport on the ridge, the height of the ridges reduced with time, disappearing after 1-2 days.

The emergence of the spheres, when the barchanoids disappeared, caused localized erosion, being visible from the scour depressions in front of the spheres and the sediment shadows behind them. The scour, formed around the spheres, maintained a high transport rate, even though the grains were partially sheltered. The same behaviour was also observed by [14] for the erosion of sand over roughness elements caused by wind.

When the ridges disappeared, the bed developed, at this point, in two manners: in Exp. 2, 3 the sediment bed was completely washed out, while in Exp. 1, 4, 5, the bed eventually stabilized to an almost constant level. The complete erosion of Exp. 2 and 3 started at the entry of the flume and quickly developed over the whole length. This is likely due to the fact, that the smooth bed below the spheres became exposed and the grains started to glide on it, even if they were completely sheltered by the spheres.

In Exp. 1, 4, 5, a bed level above the equator of the spheres was reached. Even though the bed level was essentially stable, the sediments were not completely immobile. The bed was 
in fact marked in all three cases by very intermittent movement of the grains, with only a few grains actually being pushed downstream. The intermittance is likely connected to rare and strong turbulent events. These observations indicate that threshold conditions of motion have been effectively reached, i.e. a quasi-static equilibrium has been reached at $t \sim 65-70$ hours.

\subsection{Analysis of the time evolution of roughness density}

The bed's changing roughness, which controls the erosion, can be described by analysing the evolution of the roughness density, $\lambda_{f}$. Higher values of $\lambda_{f}$ indicate an increasing presence of the roughness elements protruding from the bed with decreasing bed coverage of the fine sediment. As it can be observed in figure $3, \lambda_{f}$ increases rapidly in the first hours for all experiments, first due to the presence of eroded areas between barchanoids and then due to the formation of the ridges. For Exp. 2 and 3, the bed level reached the equator at $t=7-8 h$ below which measurements are not possible. However, in both cases, the sediment was observed visually to continue to erode, until it was completely washed out. For Exp. 1, 4 and 5 , the bed experiences an abrupt change in behavior after $t \sim 4-6 h$. The rate at which $\lambda_{f}$ varies reduces considerably and the curves approach a constant value near $t \sim 60-70 h$. The abrupt change clearly indicates a transition in the physical mechanisms acting on the sediments, changing from high erosion rates to a significant reduction in the erosive capacity. Table 3 lists the time instant $T$ and the values of the roughness density, at which the transition takes place.

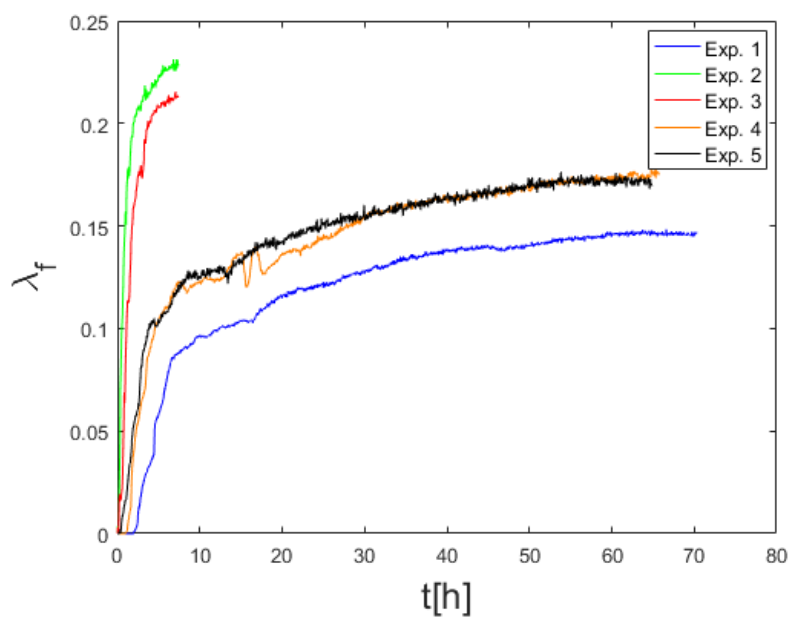

Figure 3. Temporal evolution of the roughness density $\lambda_{f}$

As seen in table 3, the transition takes place at an approximately constant roughness density, but the time instant for the transition varies between experiments. Since Exp. 1, 4 and 5 are characterized by the same shear stress, but all other flow parameters (water depth, flow rate, $\mathrm{Re}$ ) are different, it can be stated that the transitional time depends on the flow conditions.

The transitional roughness density $\lambda_{f, t}$ instead is approximatively constant for Exp. 1, 4, 5, 
for which the shear velocity is essentially constant and the AR varies. The transitional roughness density can depend therefore only on the shear velocity. Furthermore, the transition is not affected by secondary currents, since by varying the aspect ratio and maintaining constant the shear velocity, $\lambda_{f, t}$ does not vary. Unfortunately, it is not possible to determine whether a transition occurs also in the case of Exp. 2 and 3, since the measurement uncertainty above a value of $\lambda_{f}$ equal 0.2 becomes too high, since the bed level is near the equator of the spheres. Further studies are being conducted, in order to determine whether, in the presence of higher shear velocities, the transition occurs at higher roughness densities.

The fact that the roughness density reaches an almost constant value at the end of the exper-

Table 3. Roughness density and time instant of the transition

\begin{tabular}{lll}
\hline & $\mathrm{T}[\mathrm{h}]$ & $\lambda_{f, t}$ \\
\hline Exp. 1 & 7.25 & 0.086 \\
Exp. 4 & 4.1 & 0.099 \\
Exp. 5 & 4 & 0.097 \\
\hline
\end{tabular}

iments, shows that the bed is not evolving anymore and that a quasi static equilibrium of the bed has been reached. In a similar way, [3] showed that in the presence of sediment feeding a dynamic equilibrium of the system is possible, characterized by sediment transport with same erosion and deposition rates, so that the bed reaches a constant level. In the field of aeolian sediment transport, [14] show also that the roughness density tends to a constant value (as observed here), when studying the erosion of sand over a staggered array of spheres in a wind tunnel with no sand supply. The system reaches also in this case a condition characterized by a constant bed elevation and no sediment transport. The shear stress partitioning of Raupach [13] is used by [14] to explain the behaviour of the system. The same theory is applied in this study to see whether it leads to a satisfactory explanation of the experimental results.

According to the shear stress partitioning theory of Raupach [13], the portion of shear stress acting on the grains is inversely proportional to the roughness density and of the parameter $\beta$ (ratio between $C_{R}$, drag coefficient of an isolated roughness element, and $C_{S}$, drag coefficient of the surface without the presence of roughness elements). As a consequence, the higher the roughness density, the lower the shear stress acting on the sediments. The maximum value of shear stress acting on the sediments for Exp. 1,4,5 (with a final roughness density $\lambda_{f}$ of about 0.15 ) was calculated to be about $12 \%$ of the global shear stress acting on the bed (by taking $C_{R}=0.3$, as assumed by [13], and evaluating $C_{S}=\sqrt{8 / f}$, where $f$ is the friction coefficient, calculated on the basis of the formula of Marchi [26]), i.e. about $0.25 \mathrm{~cm} / \mathrm{s}$ in terms of shear velocity. Since the critical shear velocity is equal to $1.8 \mathrm{~cm} / \mathrm{s}$ (corresponding to the threshold Shields parameter $\theta_{t, u}=0.031$ ), the sediment transport is reduced, in the case of Exp. 1,4,5, under the threshold of motion, so that a quasi static equilibrium is reached. The protruding roughness elements are able to extract enough momentum from the flow, to stop sediment transport. The model predicts, for Exp. 2 and 3 (with a final roughness density $\lambda_{f}$ of 0.52 ), a value of shear stress acting on the grains equal to $8 \%$ of the total shear stress, so that no sediment transport should take place. The experiments show instead that the bed is completely washed out, so that the Raupach model wrongly predicts the effective shear stress acting on the bed. The Raupach model does not consider in fact the interaction of the roughness elements' wakes when they are closely packed, i.e. with high roughness densities, as it was also observed by [27]. [28] highlights also the fact, that the $\beta$ parameter is not a constant, as supposed by the Raupach model, but depends on the protrusion of the roughness elements. A further limitation of the model lies in the complete neglect of the wake induced turbulence, which might penetrate into the roughness layer leading to local higher shear stress values. The intermittent sediment transport at the end of the Exp. 1,4,5 sustains this hypothesis. Fur- 
ther hydrodynamic studies concerning the type of turbulence characterizing the wake of the roughness elements and interesting the roughness layer should be performed.

\section{Conclusions}

The interaction between fine sediments and a coarse immobile substrate under sediment supply limited conditions was studied. A series of flume experiments were carried out to analyse the evolution of the bed. It was observed that, first large barchanoids formed, followed by ridges, which characterized the bed topography for longer times. Depending on the total shear stress and independent of flow conditions (AR and Re), two possible outcomes of the evolution of the system were determined: full erosion or a stable bed with low intermittent sediment movement. In the latter case, an abrupt transition between a highly erosive behaviour towards an asymptotically stabilizing one was observed. The final stable state reached by the system can be considered a quasi-static equilibrium. The shear stress partitioning theory by Raupach was shown not to be able to adequately explain the results obtained in the case of high roughness density values.

\section{References}

[1] R. Kuhnle, D. Wren, E. Langendoen, J. Rigby, J. Hydraul. Eng. pp. 167-176 (2013)

[2] T. Lisle, S. Hilton, R-5 Fish Habitat Relationship Technical Bulletin 5 (1991)

[3] P. Grams, Ph.D. thesis, Johns Hopkins University (2006)

[4] E. Meyer-Peter, Müller, Proc. 2nd Meeting IAHSR pp. 1-26 (1948)

[5] F. Engelund, E. Hansen, A monograph on sediment transport in alluvial streams (1967)

[6] C.E. Zanke, Int. J. Sediment Res. 16, 1 (2001)

[7] P. Grams, P. Wilcock, Water Resources Research 43 (2007)

[8] P. Grams, P. Wilcock, J. Geophys. Res. Earth Surf. pp. 188-211 (2014)

[9] E. Yager, J. Kirchner, W. Dietrich, Water Resour. Res. 43 (2007)

[10] D. Wren, E. Langendoen, R. Kuhnle, J. Geophys. Res. 116 (2011)

[11] P. Wilcock, S. Kenworthy, Water Resources Research 38 (2002)

[12] G. Parker, P. Klingeman, D. McLean, J. of Hydr. Div. 18, 1409 (1982)

[13] M. Raupach, Boundary Layer Meteorology 60, 375 (1992)

[14] W. Nickling, C. McKenna Neumann, Sedimentology 42, 403 (1995)

[15] C. McKenna Neuman, Geomorphology 25, 1 (1998)

[16] M. Kleinhans, A. Wilbers, A. De Swaaf, J. van den Berg, Journal of Sedimentary Research 72, 629 (2002)

[17] S. McLean, Marine Geology 42, 49 (1981)

[18] I. Nezu, J. Hydraul. Eng. 131, 229 (2005)

[19] S. McLelland, P. Ashwoth, J. Best, J. Livesey, J. Hydraul. Eng. 125, 463 (1999)

[20] I. Nezu, H. Nakagawa, Turbulence in Open-Channel Flows (IAHR, 1993)

[21] R.A. Bagnold, Philos. Trans. 249, 235 (1956)

[22] S. Dey, Fluvial Hydrodynamics (Springer, Heidelberg, 2014)

[23] S. Tjerry, J. Fredsoe, J. of Geo. Res. 110, 1 (2005)

[24] E. Franklin, C. F., J. Fluid. Mech 675, 199 (2011)

[25] B. Flemming, Proceedings of Marine sandwave dynamics (2000)

[26] E. Marchi, L'Energia elettrica 42 (1961)

[27] Y. Shao, Y. Yang, Journal of Geophysical Research 113 (2008)

[28] D. Crawley, W. Nickling, Bundary Layer Meteorology 107, 445 (2003) 